\title{
Comparative Study between Addresses Compression Scenarios Applied In IPv6 Networks
}

\author{
Mustafa Bakry Hussen, Dr. Amin Babiker A/Mustafa \\ Department of Communication Engineering, Al-Neelain University
}

\begin{abstract}
IPv6 provides solutions to the problems of the growth of internet, specially to the lack of addresses, But the main point of IPv6 addressing is the 128 bits addresses, which give a wide addressing space, In this paper we worked on reducing the number of bits used in addresses to the simplest form, therefore aiming at improving the performance of the network and work to increase speed of sending and receiving data and aiming at increasing processing speed and obtaining high network performance of data.
\end{abstract}

Keywords: IPv6, IPv4, OPNET

\section{Introduction}

Today IPv6 over IPv4 tunnels are widely used to connect large regional IPv6 networks, because it is relatively hard to construct an international or cross-continent native IPv6 network. This makes the characteristics of IPv6 over IPv4 tunnels very vital to the performance of the global IPv6 Internet. Migrating from IPv4 to IPv6 in an instant is impossible because of the huge size of the Internet and of the great. Number of IPy4 users. Moreover, many organizations are becoming more and more dependent on the Internet for their daily work, and they therefore cannot tolerate downtime for the replacement of the IP protocol. As a result, there will not be one special day on which IPv4 will be turned off and IPv6 turned on because the two protocols can coexist without any problems. The migration from IPv4 to IPv6 must be implemented node by node by using auto configuration procedures to eliminate the need to configure IPv6 hosts manually. This way, users can immediately benefit from the many advantages of IPv6 while maintaining the possibility of communicating with IPv4 users or peripherals. Consequently, there is no reason to delay updating to IPv6.In this paper we are going to investigate the IPV6 and the IPV4 and when to decide to immigrate to IPV6.

The main advantage of ipv6 over ipv4 it is larger addresses space, the length of an ipv6 address is 128 bits, which give a wide addressing space, and provides solutions to the problems of the growth of internet, especially to the lack of addresses.

\section{Methodology}

OPNET Modeler Suite we test and demonstrate technology designs before production by allow for communication between real devices or software and assimilated network then analyzes networks to compare the impact of different technology designs on end-to-end behavior.

In this simulation we designed a network consists of two servers linked across internet and clients in other site and all of this devices work by ipv6 addresses, and we gave the first server IP addressee without compressed, while the other server with addresses compressed. The main objective of this simulation is observe and control the speed of the flow of data from two servers as shown in figure1, then we work compared to delay from end to, also we compared the time consumer in both IP traffic sent (packets/sec), and IP traffic received ( packets/sec).

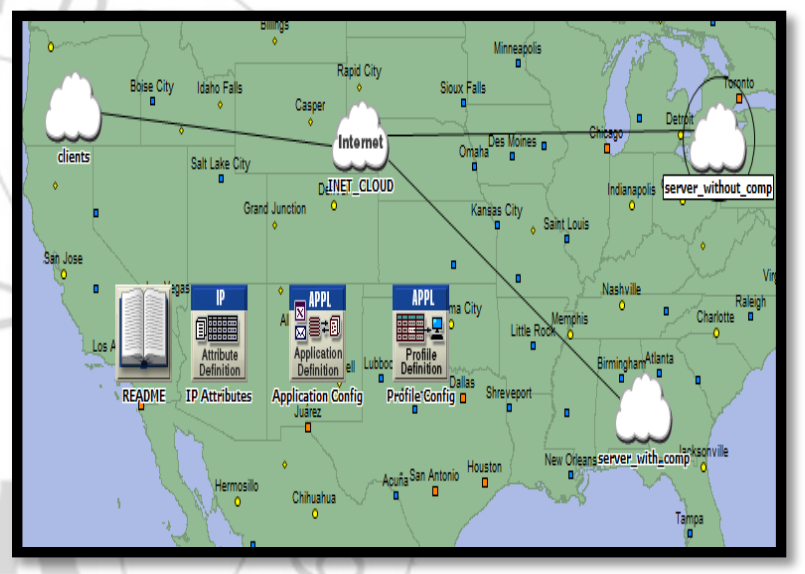

Figure 1: System Model

\section{Results}

Time Average In IP End To End Delay:

We sending data from the clients to each server and worked to run the simulation and follow-up delay in each of servers as shown in figure 2 .

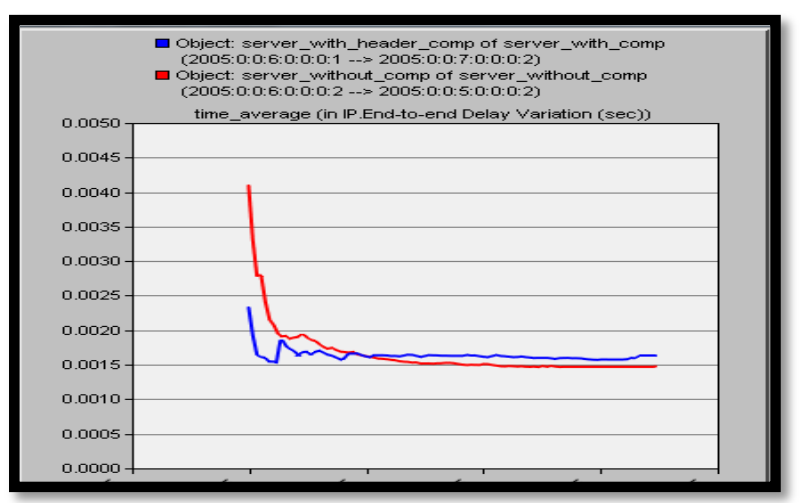

Figure 2: End To End Delay

The delay in the server are without compressed 0.0040 while the delay did not exceed 0.0023 for server with 


\section{International Journal of Science and Research (IJSR) \\ ISSN (Online): 2319-7064}

Index Copernicus Value (2013): 6.14 | Impact Factor (2015): 6.391

compressed and thus very great improvement in the performance of the network and increase speed the flow of data and get the highest output data per second. With this delay, the delay will be greater in the case of the compression but worth a few means we have won in a number of parameters, but did not lose much after the compression process.

Time Average in IP Traffic Sent (Packets/Sec):

In this simulation we compare time consumer for traffic sent (packets /sec) in each server and worked to run the simulation as shown in figure 3.

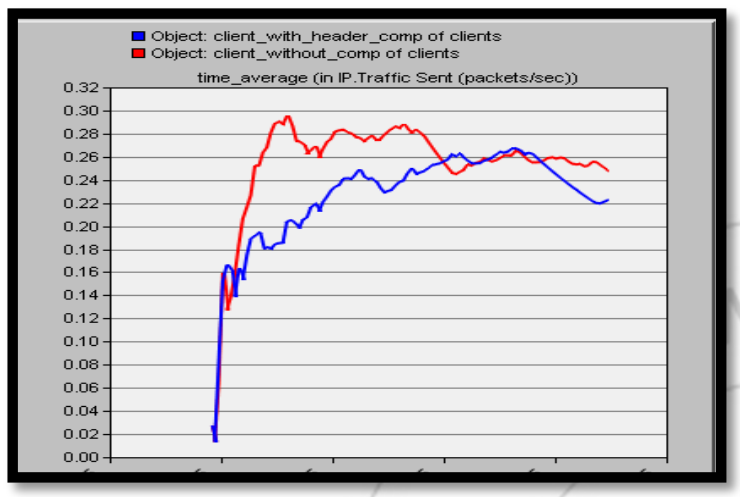

Figure 3: Traffic Sent

\section{Conclusion}

The main goal is always is the improvement of the performance of the network, we have worked in this paper improves performance by reducing the length number of bits used in ipv6 addresses by using three basic rules to pressure, the result is reduce delay from end to end and reduce the average time consuming in sent and received packets and get the highest output data per second. And we have achieved improve the performance of the network, and provide a good service for the clients over network and get a high efficiency network.

\section{References}

[1] "OPNET: IPv6 for R\&D Specialized Model" [Online] Available: http://www.opnet.com/solutions/network_rd/simulatio n_model_library/IPv6.html, Accessed on 15 December, 2013

[2] IP Version 6 Addressing Architecture, RFC 2373, R. Hinden, S. Deering, July 1998

[3] IPv6.com/articles/general/IPv6-Addressing

[4] www.ripe.net/participate/member-support/newlir/ipv6_reference_card.pdf

[5] http://www.6diss.org/workshops/.../mobility

[6] Douglas E.comer, Computer Network And Internets, Five Edition, 2008

The result for this simulation the average time for sent packets from source to destination in the server are without compressed between (0.014 to 0.29), while the average time between ( 0.01 to 0.26$)$ for server with compressed. The less time consumer in the transmitter means it grew the number of packets sent and improves network efficiency.

Time Average in IP Traffic Received (Packets/Sec):

In this simulation we compare time consumer for traffic received (packets $/ \mathrm{sec}$ ) in each server and worked to run the simulation as shown in figure 4 .

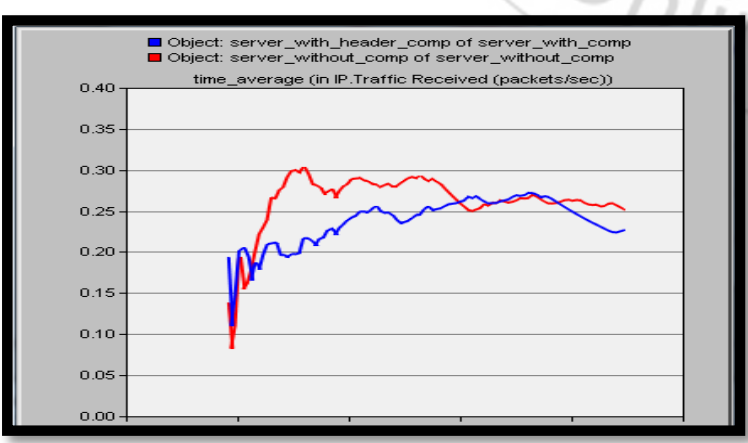

Figure 4: Traffic Received

The result for this simulation the average time for sent packets from source to destination in the server are without compressed (0. 30), while the average time (0.26) for server with compressed. We found that the data package arrived faster when compressed, but we find we have lost some of these packages.
[7] Word, Mark "Europe hits old internet address limits" BBC retrieved 15 September 2012

[8] A.Dutta, J.ALberi, A.Cheng, B.Horgan, T.McAuley, D.Chee, B.Lyles, "IPv6 Transition techniques for legacy Application": 582, MILCOM 2006. 